\title{
Upper respiratory tract infections and general anaesthesia in children
}

\author{
Peri-operative complications and oxygen saturation
}

\author{
L. LEVY, U. A. PANDIT, G. I. RANDEL, I. H. LEWIS AND A. R. TAIT
}

\begin{abstract}
Summary
Conflicting reports regarding the hazards of anaesthesia in children presenting for surgery with an upper respiratory tract infection have appeared in the literature. In the present study 130 children undergoing general anaesthesia with face mask for myringotomy and grommet insertion were graded as having either an acute or recent upper respiratory tract infection or were asymptomatic according to predetermined clinical symptoms and signs. The severity of respiratory and related complications were scored during induction, emergence and recovery. The peripheral oxygen saturation was recorded during induction, emergence, transfer to the recovery ward and in the recovery ward itself. There were no significant differences $(p>0.05)$ in the complication scores between the three groups of children. However, the incidence of hypoxaemia (oxygen saturation $\leq 93 \%)$ was significantly greater during transfer in the acute infection group $(p=0.001)$ and the recent infection group $(p=0.02)$, as well as during recovery in the acute group $(p=0.03)$ compared with asymptomatic children.
\end{abstract}

\section{Key words}

Anaesthesia; paediatric.

Infection; upper respiratory tract.

Many children scheduled to undergo myringotomy and insertion of grommet for chronic otitis media suffer from frequent upper respiratory symptoms including sneezing, rhinorrhoea, congestion and cough. These symptoms may be related to the underlying chronic pathology or may be indicative of a viral upper respiratory tract infection (URTI). In general, children presenting for elective surgery with URTIs are cancelled until asymptomatic. Normal children may suffer from an average of three to eight URTIs per year [1] and if surgery was delayed for 4 to 6 weeks after each of these episodes there might be only 4 weeks in the year when the child would be considered fit. The practice of postponing surgery for patients presenting with an URTI stems from the widely held belief that anaesthesia increases the risk of peri-operative complications. Although several studies support this viewpoint, others suggest that for certain procedures there is no increased risk for patients presenting with acute, uncomplicated URTIs [2-5]. Part of the difficulty in evaluating and comparing these studies is due to the confounding effect of variations in surgical site, tracheal intubation, patient age, upper respiratory infection criteria and lack of definition or gradation of 'respiratory complications'.
Whereas most investigations of upper respiratory tract infection and anaesthesia have focused on the incidence of rare, major complications such as complete laryngospasm, intra-operative lobar collapse and postoperative pneumonia, the presence of more common respiratory complications, such as partial airway obstruction, excessive secretions, coughing and oxygen desaturation has not been quantified. Postoperative hypoxaemia has been documented in symptomatic and asymptomatic children with pulse oximetry $[6,7]$ but possible intra-operative hypoxaemia has yet to be evaluated.

The present study was designed to determine if a child with symptoms and signs consistent with an upper respiratory tract infection undergoing mask anaesthesia for myringotomy and insertion of grommet is at increased risk for peri-operative respiratory complications or hypoxaemia.

\section{Methods}

This study was approved by the local Ethics Review Committee and informed consent obtained from a parent before anaesthesia. One hundred and thirty children

L. Levy, MD, G.I. Randel," MD, I.H. Lewis, MB, BS, MRCP, FFARCS, A.R. Tait, PhD, Assistant Professors of Anesthesiology, U.A. Pandit, MD, Associate Professor of Anesthesiology, Section of Pediatric Anesthesiology, University of Michigan, Room C4139, Box 0800, C.S. Mott Children's Hospital, Ann Arbor, Michigan, 48109, USA.

*Present address: Department of Anaesthesiology, Northwestern University, Chicago, Illinois, USA.

Correspondence should be addressed to Dr U.A. Pandit.

Accepted 10 March 1992. 
Table 1. Scoring system for each of the seven complications: minimum score $=7$, maximum score $=28$.

\begin{tabular}{|c|c|c|c|c|}
\hline & 1 & 2 & 3 & 4 \\
\hline 1. Cough & None & Occasional & Frequent & Continuous \\
\hline 2. Breath holding & None & $<15 \mathrm{~s}$ & $15-30 \mathrm{~s}$ & $>30 \mathrm{~s}$ \\
\hline 3. Airway obstruction (relieving factors) & None & $\begin{array}{l}\text { Partial } \\
\text { (reposition } \\
\text { airway) }\end{array}$ & Partial (CPAP) & Complete (muscle relaxant) \\
\hline 4. Bronchospasm & None & Expiration only & $\begin{array}{l}\text { Inspiration } \\
\text { Expiration }\end{array}$ & Difficult to ventilate \\
\hline 5. Secretions & None & $\begin{array}{l}\text { Minimal no } \\
\text { suction }\end{array}$ & $\begin{array}{l}\text { Moderate } \\
\text { suction } \times 1\end{array}$ & Copious suction $>1$ \\
\hline 6. Excitement & None & $<30 \mathrm{~s}$ & $30-60 \mathrm{~s}$ & $>60 \mathrm{~s}$ \\
\hline 7. Nausea, vomiting or regurgitation & None & Nausea & Retching & Vomiting or regurgitation \\
\hline
\end{tabular}

conforming to ASA grades 1 or 2, undergoing myringotomy and insertion of grommet were studied. Children were placed into one of three groups based upon their presenting symptoms: group 1, acute URTI present; group 2, URTI within the last 2 weeks but asymptomatic on the day of operation and group 3, no URTI symptoms for more than 2 weeks. Upper respiratory tract infection was defined according to the criteria used by Tait and Knight [8]. The presence of two or more of the following symptoms were required (reported by parent or child): (1) sore or scratchy throat, (2) sneezing, (3) rhinorrhoea, (4) congestion, (5) malaise, (6) nonproductive cough, (7) fever less than $101^{\circ} \mathrm{F}$ or (8) laryngitis. Combinations of (1) and (5), (2) and (3), (3) and (6), or (4) and (6) required the presence of one additional symptom. Children with a fever greater than $101^{\circ} \mathrm{F}$, productive cough or evidence of a lower respiratory tract infection were not studied.

The children received no premedication and a baseline oxygen saturation was obtained before induction of anaesthesia with the patient breathing room air. Anaesthesia was induced with $66 \%$ nitrous oxide in oxygen followed by $1-3 \%$ halothane by face mask and Mapleson D system. During surgery all children continued to breathe spontaneously the same concentrations of nitrous oxide and oxygen, with up to $2 \%$ halothane. At the conclusion of the procedure $100 \%$ oxygen was administered for $3 \mathrm{~min}$ following the discontinuation of the anaesthetic agent and immediately before transfer to the recovery ward. The electrocardiogram, blood pressure (Dinamap) and peripheral oxygen saturation (Nellcor N-100 pulse oximeter) were monitored during induction, maintenance and emergence from anaesthesia.

A blinded observer scored the induction, emergence and recovery periods for the incidence and severity of the following complications: (1) airway secretions, (2) coughing, (3) breath holding, (4) bronchospasm, (5) upper airway obstruction including laryngospasm, (6) excitement or restlessness and (7) vomiting or regurgitation. These complications were each rated on a four-point scale and then pooled to obtain a total score. For example, airway obstruction was rated as either (1) absent, (2) partial, (3) partial, requiring positive pressure ventilation to overcome or (4) total, requiring muscle relaxation with or without intubation. The total complication score could therefore range from a minimum of 7 to a maximum of 28 (Table 1 ). Oxygen saturation was recorded by the same blinded observer every minute during induction, emergence and transport to the recovery room, and was subsequently recorded every minute for the first $5 \mathrm{~min}$ in the recovery room followed by every $5 \mathrm{~min}$ for the next $15 \mathrm{~min}$. Supplemental oxygen was given immediately in the recovery room if the oxygen saturation fell below $90 \%$. The duration of surgery, anaesthesia, transfer from the operating room to the recovery room and time to discharge (taken from the end of anaesthesia to discharge from hospital) were also recorded.

Continuous data were analysed using analysis of variance (ANOVA). Analysis of categorical variables was performed using a contingency table (expanded Chi-square) and the Bonferroni correction was applied when multiple group comparisons were made. A p value of $<0.05$ was considered statistically significant.

\section{Results}

One hundred and thirty children were studied; 22 fulfilled the criteria for the acute URTI group, 28 the recent URTI group and 80 the asymptomatic group. There were no statistically significant differences in age or sex distribution between the acute infection, recent infection or asymptomatic groups (Table 2). There were also no differences between the groups in terms of the duration of surgery, anaesthesia and transfer or the time to discharge (Table 2). None of the children received atropine.

There were no significant differences in the complication scores during induction, emergence or at any time during the recovery period before discharge from hospital (Table 3). There were also no significant differences in the

Table 2. Demographic and perioperative data. Mean (SD or \%).

\begin{tabular}{lccc}
\hline & Acute URTI & Recent URTI & Asymptomatic \\
\hline Age; months & $47.2(44.7)$ & $29.0(26.4)$ & $46.1(40.3)$ \\
Weight; kg & $17.7(10.0)$ & $13.3(6.1)$ & $16.9(9.4)$ \\
Sex (M) & $15(68)$ & $19(68)$ & $51(64)$ \\
$\quad$ (F) & $7(32)$ & $9(32)$ & $29(36)$ \\
Duration of surgery; min & $8.6(3.9)$ & $8.9(3.5)$ & $9.7(4.4)$ \\
Duration of anaesthesia; min & $15.0(5.3)$ & $15.3(5.0)$ & $16.2(5.5)$ \\
Duration of transport; s & $88.0(26.0)$ & $95.0(33.0)$ & $89.0(41.0)$ \\
Time to discharge; min & $65.9(14.3)$ & $66.2(13.4)$ & $66.5(17.6)$ \\
\hline
\end{tabular}


Table 3. Complication scores at induction, emergence and recovery. Mean (range).

\begin{tabular}{lcccc}
\hline & $\begin{array}{c}\text { Acute URTI } \\
(n=22)\end{array}$ & $\begin{array}{c}\text { Recent URTI } \\
(n=28)\end{array}$ & $\begin{array}{c}\text { Asymptomatic } \\
(n=80)\end{array}$ & $\mathrm{p}$ \\
\hline Induction & $7.9(7-15)$ & $8.5(7-12)$ & $8.0(7-12)$ & 0.16 \\
Emergence & $7.6(7-10)$ & $7.6(7-12)$ & $7.6(7-12)$ & 0.52 \\
Recovery & $8.8(7-15)$ & $8.7(7-12)$ & $8.6(7-15)$ & 0.91 \\
\hline
\end{tabular}

Table 4. Number (\%) of children with scores of seven (no complication) at induction, emergence or recovery.

\begin{tabular}{lcccc}
\hline & $\begin{array}{c}\text { Acute URTI } \\
(n=22)\end{array}$ & $\begin{array}{c}\text { Recent URTI } \\
(n=28)\end{array}$ & $\begin{array}{c}\text { Asymptomatic } \\
(n=80)\end{array}$ & $\mathrm{p}$ \\
\hline Induction & $12(55)$ & $10(36)$ & $36(45)$ & 0.41 \\
Emergence & $13(59)$ & $16(57)$ & $62(77)$ & 0.06 \\
Recovery & $7(14)$ & $10(20)$ & $32(65)$ & 0.76 \\
\hline
\end{tabular}

incidence of children with scores of 7 (no complication) at induction, emergence or recovery (Table 4). Total laryngospasm requiring intubation or muscle relaxants did not occur. One child in the asymptomatic group developed laryngospasm which was successfully treated with CPAP (continuous positive airway pressure) applied by means of the mask. Mild, partial laryngospasm occurred in 10 patients during induction (four with acute infection, one with recent infection and five asymptomatic cases) three patients during emergence (all in the asymptomatic group) and three patients in the immediate recovery period (all in the asymptomatic group). None of these findings were statistically significant.

The oxygen saturation did not differ significantly between groups before or during induction of anaesthesia (Table 5). However, there were significant differences between the three groups during transfer and recovery (Table 5). The incidence of hypoxaemia (oxygen saturation $\leq 93 \%$ ) was significantly greater during transfer in the acute infection group $(\mathrm{p}=0.001)$ and the recent infection group ( $p=0.02)$, as well as during recovery in the acute group $(p=0.03)$ compared with asymptomatic children. Hypoxaemia during transfer did not appear to depend on the duration of transfer or the age of the child. Twenty-one children in our study were under one year of age (15, asymptomatic; four, recent URTI; two, acute URTI) and 35 were over 5 years of age (24, asymptomatic; three, recent URTI; eight, acute URTI); none of these patients had a saturation less than $94 \%$ during transfer. We were unable to record oxygen saturations on six children during transfer due to excessive movement causing interference with the pulse oximeter (one, acute URTI; one, recent URTI; four, asymptomatic) and their data were excluded from analysis (Table 4). There were no complications other than those reported; no child required hospitalization and there were no significant differences in discharge times between the three groups.

\section{Discussion}

The management of the patient presenting with an upper respiratory tract infection for elective surgery is a perennial clinical dilemma. Despite the medical, social and financial implications of delaying treatment few studies have addressed this issue. Some of these are anecdotal or poorly controlled and comparisons are hindered by differences in study design.

Part of the controversy surrounding anaesthetic risk and upper respiratory tract infection is due to the problem of defining when such a condition is present. Confirmatory evidence of an URTI should ideally include viral cultures with acute and convalescent antibody titres, but this is clearly neither practical nor cost-effective for a self-limiting, usually benign illness. In a previous study by Tait and Knight [3] nasopharyngeal cultures were taken from a random sample of symptomatic and asymptomatic children. They confirmed the presence of virus in about $25 \%$ of cases overall, although this is likely to be an underestimate. Consequently, most papers describing the management of the patient with upper respiratory symptoms must base their definition on the presence or absence of clinical symptoms. Unfortunately, there are no unique clinical criteria for the diagnosis of upper respiratory tract infections and they may be difficult to distinguish from some allergic conditions. The criteria used in our study have been used previously and attempt to identify the types of symptom that are routinely used by clinicians to make a decision regarding the patients' status [8]. These criteria, together with information obtained from the child's parents as to the nature of the symptoms, were used to categorise the patient into one of three groups.

There are few previous investigations designed to determine if children with an upper respiratory tract infection are at increased risk of peri-operative respiratory complications. In 1979, McGill et al. [2] reported 11 cases of pulmonary dysfunction following general anaesthesia. Coarse breath sounds, despite suctioning and radiographic

Table 5. Minimum oxygen saturation during induction, transfer and recovery: incidence (\%).

\begin{tabular}{|c|c|c|c|c|}
\hline & Acute URTI & Recent URTI & Asymptomatic & $\mathrm{p}$ \\
\hline \multicolumn{5}{|l|}{ Induction } \\
\hline$\geq 94 \%$ & $21(95)$ & $26(93)$ & $76(95)$ & 0.89 \\
\hline$\leq 93 \%$ & $i(5)$ & $2(7)$ & $4(5)$ & \\
\hline \multicolumn{5}{|l|}{ Transfer } \\
\hline$\geq 94 \%$ & $15(71)^{*}$ & $24(89) \dagger$ & $75(99)$ & 0.001 \\
\hline$\leq 93 \%$ & $6(29)$ & $3(11)$ & 1 (1) & \\
\hline \multicolumn{5}{|l|}{ Recovery } \\
\hline$\geq 94 \%$ & $15(68)$ & $21(75)$ & $72(90)$ & 0.02 \\
\hline$\leq 93 \%$ & $7(32)$ & $7(25)$ & $8(10)$ & \\
\hline
\end{tabular}

*Acute vs asymptomatic $p=0.001$; †recent vs asymptomatic $p=0.02$; 9 acute vs asymptomatic $p=0.03$ (contingency table with Bonferroni correction). 
evidence of atelectasis or infiltrate were noted in 10 children who gave a history of respiratory infection during the 4 weeks before surgery. A retrospective study by Tait and Knight [8] of 3585 children with acute, recent or no upper respiratory infection undergoing various operative procedures found a small but significant increase in the prevalence of respiratory complications (laryngospasm, bronchospasm, breath-holding, stridor) in the recent infection (within 2 weeks) group. In a follow-up prospective study, the same authors [3] investigated 489 children undergoing myringotomy under general anaesthesia with a face mask. This time there were no significant differences in complications (laryngospasm, bronchospasm, apnoea or arrhythmias) between children with an acute, recent or no infection, although oxygen saturations were not recorded. These latter findings confirm those of the present study. In contrast, however, Cohen and Cameron [4] examined a large paediatric database which was prospectively obtained from 1982 to 1987 and found that children with an upper respiratory infection were 2 to 7 times more likely to experience 'respiratory related adverse events' during the intra-operative, recovery and postoperative phase. Furthermore, these risks were increased by tracheal intubation.

Some of the differences between these studies may be explained by several factors. Firstly, some of the patients in the study by Cohen and Cameron [4], the majority of patients in Tait and Knight's earlier retrospective study [8], and all of the patients in McGill's case reports [2] were intubated for their procedures. Bypassing the nasopharynx and orpharynx with high flows of dry gases encourages the inspissation of secretions leading to mucus plugging and atelectasis. This effect may be more pronounced with longer, major procedures. Tracheal intubation may also lead to, or exacerbate, intraluminal swelling of the trachea which can have significant physiological implications for the paediatric patient [5]. Secondly, the earlier studies by Tait and Knight [4] and McGill et al. [2] are retrospective. The study by Cohen and Cameron is strengthened by the size of their database $(n=29220)$ but the upper respiratory tract infection was not defined and there was scanty information concerning the timing of the infection relative to the date of the surgical procedure. None of these investigations included data on peripheral oxygen saturation and all lacked a uniform anaesthetic regimen.

One previous study has investigated peri-operative hypoxaemia in children with upper respiratory infections. DeSoto and coworkers [9] found that children with clinical symptoms of infection have a different pattern of oxygen saturation compared to asymptomatic children. These workers found a significantly higher incidence of transient hypoxaemia (oxygen saturation $<95 \%$ ) in the recovery room in children with signs of respiratory infection at the time of surgery or in the preceding week compared to asymptomatic children.

In the present study we were able to demonstrate that children with an acute upper respiratory tract infection have a higher incidence of hypoxaemia (oxygen saturation $\leq 93 \%$ ) during transfer and recovery. Children who developed hypoxaemia during transfer tended to continue desaturating in the immediate recovery period. During this investigation supplemental oxygen was given only if the peripheral oxygen saturation fell below $90 \%$; all such children responded with a rapid rise in oxygen saturation.
None of the children in the study by DeSoto and colleagues [9], or the present study, developed postoperative respiratory complications or required a prolonged hospital stay.

In a study of hypoxaemia after general anaesthesia in healthy children, Motoyama and Glazener [7] found the mean peripheral oxygen saturation on arrival in recovery to be $93 \%$ (corresponding to a calculated arterial oxygen tension of approximately $66 \mathrm{mmHg}$ ). This figure was significantly different from the pre-operative mean of $97.6 \%$. These authors suggested that supplementary oxygen should be given in the postoperative period at least until the child is awake enough to reject it. We chose to define hypoxaemia as $\leq 93 \%$ because of the findings by Motoyama and Glazener [7] and because we felt that supplemental oxygen should normally be given at this point before further desaturation (corresponding to the steep part of the oxygen-haemoglobin dissociation curve) occurred.

An editorial in the British Journal of Anaesthesia [10] suggested that there was little evidence to show that anaesthesia in adult patients with upper respiratory infection predisposed to respiratory complications. Our data suggest that children with an acute or recent upper respiratory infection have an increased likelihood of transient hypoxaemia in the peri-operative period, which responds promptly to supplemental oxygen therapy. We believe that minor surgery not requiring tracheal intubation can proceed in children who have acute or recent infection and who are not toxic. Children with a fever $>101^{\circ} \mathrm{F}$, productive cough or evidence of a lower respiratory tract infection were not studied. We advocate continuous monitoring with pulse oximetry during the peri-operative period and the routine use of supplemental oxygen therapy during both transfer and recovery

\section{Acknowledgments}

The authors thank Nellcor for the loan of a Nellcor N-100 pulse oximeter for the duration of this study.

\section{References}

[1] BERry, FA. The child with the runny nose. Anesthetic management of difficult and routine pediatric patients. New York: Churchill Livingstone, 1986: 349-67.

[2] MCGill WA, Coveler LA, EPSTEIN BS. Subacute upper respiratory infection in small children. Anesthesia and Analgesia 1979; 58: 331-3.

[3] TAIT AR, KNIGHT PR. The effects of general anesthesia on upper respiratory tract infections in children. Anesthesiology 1987; 67: $930-5$.

[4] Cohen MM, Cameron CB. Should you cancel the operation when a child has an upper respiratory tract infection? Anesthesia and Analgesia 1991; 72: 282-8.

[5] Koka BV, Jeon IS, Andre JM, Mackay I, Smith RM. Postintubation croup in children. Anesthesia and Analgesia 1977; 56: 501-5.

[6] Kataria BK, Harnik E, Mitchard R, Kim Y. Ahmed S. Postoperative arterial oxygen saturation in the pediatric population during transportation. Anesthesia and Analgesia 1987; 66: S96.

[7] Motoyama EK, Glazener CH. Hypoxemia after general anesthesia in children. Anesthesia and Analgesia 1986; 65: 267-72.

[8] TAIT AR, KNIGHT PR. Intraoperative respiratory complications in patients with upper respiratory tract infections. Canadian Journal of Anesthesia 1986; 34: 300-3. 
[9] DeSoto H, Patel RI, Soliman IE, Hanallah RS. Changes in oxygen saturation following general anesthesia in children with upper respiratory infections signs and symptoms undergoing otolaryngological procedures. Anesthesiology 1988; 68: 276-9.
[10] Fennelly ME, Hall. GM. Anaesthesia and upper respiratory tract infections - a non-existent hazard? British Journal of Anaesthesia 1990; 64: 535-6. 